\title{
La difusión de la ciencia en Angola a través de revistas científicas: una alternativa de mejoramiento del proceso investigativo
}

\author{
Eurico Wongo Gungula ${ }^{1}$; Wileidys Artigas $^{2}$; Arnaldo Faustino ${ }^{3}$
}

Recibido: 22 de mayo de 2020 / Aceptado: 5 de octubre de 2020

Resumen. Es indiscutible y positivo el interés que la comunidad científica internacional atribuye en la formación de la nueva generación de profesionales para la investigación científica. Angola, siendo un país signado por diversos conflictos civiles; se ha interesado en los últimos años por formar investigadores que logren que la educación universitaria avance y posicione el país en los diversos rankings académicos internacionales. En este sentido, algunas instituciones han logrado la creación de revistas científicas, lo que conlleva a la formación en diversos procesos investigativos de su personal docente. El objetivo del artículo es examinar las revistas científicas angolanas que se encuentran registradas en el portal ISSN internacional y su situación actual como alternativa de mejoramiento del proceso investigativo de las instituciones. Para esto, se hizo una revisión en el portal ISSN internacional para identificar las revistas científicas registradas y vinculadas a instituciones angolanas. Posteriormente se realizó una revisión de estas en sus páginas web, bien como las instituciones que las editan como complemento del proceso. Los resultados alcanzados indican que, la intención en la creación de revistas científicas existe, sin embargo, hay insuficiente estímulo por parte de las instituciones encargadas por el mejoramiento de los indicadores de ciencia de Angola, y con esto, lograr el posicionamiento de sus revistas en el escenario internacional.

Palabras Claves: Investigación Científica; Revistas Científicas; Difusión de la Ciencia; Proceso Investigativo; Angola.

\section{[en] The dissemination of science in Angola through scientific journals: an alternative of improving the research process.}

Abstract. The interest that the international scientific community attributes in the training of the new generation of professionals for scientific research is indisputable and positive. Angola, being a country marked by various civil conflicts; in recent years, it has been interested in training researchers to advance

1 Universidade Óscar Ribas, Luanda-Angola. Rector y Editor de la Revista Sapientiae, editada por la Universidade Óscar Ribas (UÓR).

E-mail: euricowongowongo@gmail.com

2 Universidad del Zulia, Maracaibo-Venezuela y Universidade Óscar Ribas, Luanda-Angola. Editora de la Revista Telos: Revista de Estudios Interdisciplinarios en ciencias sociales, editada por la Universidad Dr. Rafael Belloso Chacín.

E-mail: wileidys@hotmail.com; wileartigas@gmail.com

3 Universidade Óscar Ribas, Luanda-Angola. Vicerrector para los Asuntos Científicos y de Extensión Universitaria.

E-mail: arnaldo.faustino19@gmail.com 
university education and position the country in various international rankings. In this sense, some institutions have managed to create scientific journals, which leads to training in various investigative processes for in their teaching staff. The objective of the article is to examine the Angolan journals that are registered in the international ISSN portal and their current situation as an alternative to improve the institutions' investigative process. For this, a review was made on the international ISSN portal to identify the journals registered and linked to Angolan institutions. Subsequently, a review of these was carried out on their web pages, as well as the institutions that edit them as a complement to the process. The results achieved indicate that the intention in the creation of scientific journals exists, however, there is insufficient encouragement on the part of the institutions in charge of improving the science indicators of Angola, and with this, achieve the positioning of their journals in the international scenary.

Keywords: Scientific Research; Scientific Journals; Dissemination of Science; Research Process; Angola.

Sumario. 1. Introducción. 2. Contexto angolano de producción científica. 3. Alternativas para el avance de la investigación científica en el contexto angolano: la creación de revistas como una de ellas. 4. Métodos. 5. Resultados y discusión. 6. Conclusiones. 7. Referencias bibliográficas.

Cómo citar: Wongo Gungula, E.; Artigas, W.; Faustino, A. (2020) La difusión de la ciencia en Angola a través de revistas científicas: una alternativa de mejoramiento del proceso investigativo, en Revista General de Información y Documentación 30 (2), 357-377.

\section{Introducción}

El perfeccionamiento de la investigación científica en el contexto angolano es un proceso que, para comprenderlo como tal, se requiere del análisis de sus diferentes tendencias de desarrollo, fragilidades, estrategias y el impacto del acelerado desarrollo tecnológico actual en su transformación, rumbo a la calidad. Los aspectos anteriores ganan sentido y significado en las distintas Instituciones de Educación Superior (IES) angolanas, y se encuentran estudios particulares al respecto, como el caso de la Universidade Óscar Ribas (UÓR), donde estos procesos han contribuido al perfeccionamiento de las estrategias de formación de profesionales comprometidos con la construcción de conocimientos científicos significativos para la solución de problemas sociales (Faustino; Gungula; Craib, 2015; Faustino; Pérez Sánchez; Gungula, 2017).

Esto no quiere decir, que la problemática en discusión sea nueva en Angola. Lo que se pretende destacar, es la necesidad de elevar el nivel de concreción práctica, ya que, en las estrategias y políticas de investigación científica elaboradas por el Ministerio de Educación Superior, Ciencia, Tecnología e Innovación, se encuentran latentes (PDN, 2018-2022; Ministério da Economia e Planeamento, 2018).

Se trata entonces de capacitar profesionales altamente comprometidos con el desarrollo humano, sensibilizados con su contexto socioeconómico, así como, promover la ciencia, la investigación científica y tecnológica (...) (Constituição da República de Angola, Assembleia Nacional, 2010).

En este sentido, la experiencia sistematizada en la elaboración de trabajos científicos y en la publicación de sus resultados, evidencia la necesidad de desarrollar nuevas habilidades investigativas, a través de cursos de metodología de investigación científica, así como, talleres de tesis y actividades teórico-prácticas, 
resultantes de las distintas disciplinas y unidades curriculares. Siendo preciso subrayar que las actividades mencionadas constituyen una condición necesaria, pero no suficiente, en el perfeccionamiento de la investigación científica en las distintas IES angolanas en general, en busca del aumento cualitativo y cuantitativo de la producción científica institucional y visibilidad en el escenario nacional e internacional.

Se encuentran, además, condicionadas por la comprensión contextualizada del desarrollo científico y tecnológico que ocurre a nivel global, por las necesidades específicas del contexto socioeconómico y sustentable; incluida la flexibilidad en adaptar los resultados obtenidos a las distintas normas editoriales de los medios donde se pretende difundir el conocimiento científico (Chirino-Ramos, 2012; Calderón; Marshal França, 2018).

En este contexto, resulta esencial destacar, que la dificultad en estimular la producción científica de investigadores angolanos y su publicación en medios de prestigio internacional constituye actualmente una barrera que todavía no se ha logrado superar, debido a la limitada comprensión e importancia que se atribuye a nivel institucional y nacional (Gungula; Faustino; Pérez Ugartemendía, 2013).

Para contribuir a transformar dicha percepción, casi generalizada en Angola, es fundamental que se siga perfeccionando el proceso docente-educativo, a través de la contextualización de las estrategias y políticas de investigación científica, enfatizándose los procesos de evaluación del desempeño docente, del investigador científico, así como, los procesos de evaluación interna y externa de los resultados producidos por las distintas IES (Tauchen; Mendes; Catia, 2015; Silva, 2016).

La articulación e integración de los aspectos anteriores puede contribuir a una mejor adaptación a los cambios que la sociedad exige en relación con la mejoría de la calidad del proceso investigativo, así como, de las estrategias de desarrollo socioeconómico, científico, tecnológico y cultural de Angola (SNCTI, 2011; PNCTI, 2011; PLANCTI, 2014-2015; Gungula; Dieguez; Pérez Ugartemendía, 2015). En este sentido, las instituciones gubernamentales han logrado un gran adelanto al impulsar y promocionar la importancia de la mejora de la calidad de las IES, especialmente en cuanto a los procesos de investigación científica, de manera que Angola pueda posicionarse en los rankings académicos internacionales, que es una de las metas establecidas para los próximos años. Del mismo modo, también se ha iniciado la implementación del estatuto de carrera del docente universitario, que empieza a solicitar el aumento de la productividad de los actores involucrados en la docencia/investigación en las distintas IES para efectos de progresión.

Por ello, los docentes e investigadores deben desarrollar una serie de competencias que favorezcan la visibilidad de la calidad de los procesos de producción científica, en busca del desarrollo sustentable y del mejor posicionamiento de sus instituciones, destacándose en la procura de soluciones impuestas por el acelerado desarrollo científico y tecnológico actual (Tessarini; Saltorato, 2018). Esto se ha observado, de forma insuficiente, en las estrategias de desarrollo de algunas IES angolanas, al impulsar la creación de revistas científicas acorde a la importancia de estas en el ámbito nacional e internacional, para la promoción de la docencia y la investigación. 
Artigas; Gungula (2020) ya habían presentado los resultados de una investigación que procuró demostrar la cantidad de revistas científicas electrónicas disponibles en Luanda (capital de Angola), sin embargo, las mismas no se encontraban debidamente estructuradas y actualizadas. En este contexto, se originó la investigación actual en donde se buscaron otras vías de identificación de las revistas científicas electrónicas registradas internacionalmente, en aras de presentar a la comunidad científica nacional e internacional, una visión más amplia de las revistas científicas electrónicas existentes en toda la nación.

Por lo cual, se hace importante examinar las revistas científicas angolanas que se encuentran registradas en el portal ISSN internacional y su situación actual, como alternativa de avance del proceso investigativo de las distintas instituciones; de manera que, se pueda observar como a través de la creación y mantenimiento de las revistas, se puede lograr otra forma del mejoramiento del proceso investigativo nacional. Para esto, se describirá el contexto angolano en cuanto a la producción científica, se presentarán las revistas científicas y su situación actual, concluyendo acerca de los avances al respecto en el ámbito nacional e internacional.

\section{Contexto angolano de producción científica}

Capacitar y formar el sujeto social, dinámico y consciente, siempre ha sido un tema de intranquilidad en diversos contextos históricos sociales. Al respecto, se han desarrollado muchas ideas, se han sumado significativos esfuerzos, se han diseñado políticas globales, continentales, regionales, nacionales e institucionales; sin embargo, la preocupación sigue latente en muchas sociedades subdesarrolladas y en algunas desarrolladas (Fuentes; Montoya; Fuentes, 2011).

En la Constitución de la República de Angola, se encuentran distintos artículos enfocados al perfeccionamiento de la formación científica cualificada de sus ciudadanos, al incentivo de la innovación tecnológica, al estímulo del emprendimiento, a la mejoría del desempeño de los trabajadores, de las instituciones, así como, de empresas productivas en los diversos aspectos y sectores (Constituição da República de Angola, Assembleia Nacional, 2010). Se observa entonces, como en el caso de Angola, desde su principal documento legal se impulsa la formación científica.

A los aspectos mencionados, se incorpora la necesidad de comprender el rol de la capacitación permanente, como elemento llave en la gestión de procesos, bien sea a través de un proceso didáctico, pedagógico, metodológico, científico, tecnológico, como desde lo administrativo, político y socioeconómico. En este sentido, urge la elaboración de estrategias que reinterpreten el contexto formativo de los docentes e investigadores angolanos, a través de cursos de capacitación especializados y de extensión universitaria, para que puedan enfrentar los desafíos de construcción y reconstrucción de conocimientos que contribuyan para el desarrollo de la nación.

Esto quiere decir que, sin la indagación teórica de los diversos procesos y fenómenos, capaces de explicar las causas de la baja producción científica, no es 
posible desarrollar nuevas formas de pensamiento que contribuyan a revertir la situación actual, a corto y medio plazo (Sousa; Rodrigues; Pedro; Manuel, 2018). Por tanto, relacionar el tema de la producción científica con el propio aprendizaje teórico que se lleva a cabo a través de dicha producción es fundamental, pues, mientras se aprende a investigar se aprende también a conocer y reconocer los diversos fenómenos estudiados.

Así, el establecimiento de una sociedad de conocimiento, a través de la inserción de la ciencia, la tecnología y la innovación en la estrategia de desarrollo nacional, constituye un factor determinante en la edificación de sociedades económicamente competitivas, estructuradas para el desarrollo sustentable, para el combate a la pobreza y la sistematización de nuevos conocimientos (PNCTI, 2011; ENCTI, 2011).

El reconocimiento de los insuficientes resultados científico-tecnológicos, enfocados al desarrollo sustentable de Angola, debido a falta de grupos de investigadores calificados y de centros de excelencia en investigación científica asociados a IES y sectores productivos, es un indicador de la limitada inversión financiera, en busca del aumento de la producción científica y difusión de sus resultados en medios de prestigio internacional (Artigas; Gungula, 2020). Para cambiar la situación, el Estado Angolano, ha elaborado nuevos instrumentos para estimular la investigación científica y la publicación de los resultados obtenidos en distintas áreas del conocimiento, mediante políticas de progresión en la carrera docente (MESCTI, 2018c; MESCTI, 2018d).

Loa referidos instrumentos, establecen las reglas para la estructuración, organización y funcionamiento de la carrera del personal docente que realiza actividad en las IES públicas, público-privadas y privadas integradas en el subsistema de educación superior en Angola. En estas reglas se demuestra el rol del proceso investigativo como primordial para el avance en la carrera docente.

Con el mismo propósito, se han aprobado igualmente, los estatutos remuneratorios del docente universitario y del investigador científico, en aras de estimular el perfeccionamiento de los procesos de formación, de investigación científica y de extensión universitaria realizados en las IES públicas, fundamentalmente (MESCTI, 2018c; MESCTI, 2018a; MESCTI, 2018e).

Del mismo modo, en el Plan de Desarrollo Nacional - PDN (2018-2022), se observa que el objetivo estratégico 3 , del programa 1.2.7, que trata de la mejoría de la calidad de la educación superior y desarrollo de la investigación científica y tecnológica es:

Desarrollar el potencial humano, científico y tecnológico nacional, a través de la consolidación del Sistema Nacional de Ciencia y Tecnología; de la capacitación de los investigadores; de la promoción y articulación entre las Instituciones de Investigación Científica; de las IES, así como, de la creación de la Academia de Ciencias de Angola, (Plano de Desenvolvimento Nacional - PDN, 2018-2022: 85).

Sin embargo, el proceso apenas está iniciando, la producción científica en Angola sigue siendo baja y la educación superior enfrenta en la actualidad significativas 
fragilidades provocadas por la dispersión de profesionales con la misma especialización científica en distintas IES y provincias; por la débil coordinación y asesoría en materia de investigación científica por parte de las instituciones responsables por la mejoría de ese proceso; por el insuficiente número de grupos de investigación científica con grado de maestrías y doctorados; así como, por la escasez de infraestructuras universitarias y de investigación científica debidamente equipadas (PNCTI, 2011; ENCTI, 2011; PLANCTI, 2014-2015; MESCTI, 2018b; MESCTI, 2019b).

Autores como: Liberato (2014), SEES (2015), André (2016), Gungula; Faustino (2018), MESCTI (2018b), coinciden que el insuficiente número de docentes con grado de maestría y doctorados en Angola conlleva a una escasa participación institucional en la difusión de resultados, en actividades de investigación científica relevantes, tanto a nivel nacional, como internacional. Sin embargo, se ha observado interés individual de parte de los docentes en seguir capacitándose, aprovechando oportunidades en cuanto a los convenios firmados con países como Cuba y Portugal fundamentalmente, para realizar estudios avanzados, lo cual también contribuye a que los docentes solidifiquen sus conocimientos y se apropien de metodologías contextualizadas para la difusión de resultados que son exigidos en dichos programas, como requisitos para poder obtener el correspondiente grado científico.

Por otro lado, se considera que, para el perfeccionamiento del proceso de producción científica en Angola, se debe dedicar una particular atención, a la articulación de los procesos de docencia, investigación y extensión, una triada que sustenta la educación superior contemporánea, pues, en ella se asume el pleno sentido de la generación, transferencia, difusión y aplicación del conocimiento, como alternativa para impulsar el desarrollo de la ciencia, el cambio tecnológico y la innovación (Fuentes; Montoya; Fuentes, 2011; Silva, 2016; MESCTI, 2018c; MESCTI, 2018d).

En este sentido, se deben elaborar estrategias enfocadas a la capacitación especializada de los docentes e investigadores, para que estos respondan a las demandas sociales, a través de la docencia de calidad, de la investigación científica relevante, de la extensión universitaria contextualizada, de la internacionalización de los procesos, de la reforma de los sistemas de educación superior y la educación virtual (Teta, 2009; Didod Aupetit, 2014).

Para lograr mayor articulación de los aspectos mencionados y asumir nuevos retos científicos, las distintas IES angolanas necesitan consolidar la capacidad científica y tecnológica de sus recursos humanos; mejorar la calidad de sus procesos sustantivos; aumentar la inversión en proyectos de investigación, de desarrollo e innovación; así como fortalecer la cooperación nacional e internacional, para adaptarse a los cambios regionales y globales (PNCTI, 2011; ENCTI, 2011; Liberato, 2014; PLANCTI, 2014-2015).

De modo contrario, no se lograría eliminar los factores críticos que en el contexto angolano de producción científica no se deben ignorar, tales como: 
La precariedad de muchas instalaciones, la escasez de equipos de laboratorio, la docencia en varias IES públicas y privadas, la calidad cuestionable de enseñanza-aprendizaje reforzada por la escasa relación entre la teoría y la práctica, la insipiencia de la investigación científica y la existencia de una cultura de plagio, son factores críticos que limitan el desarrollo del Subsistema de Educación Superior, de investigación científica y de transferencia tecnológica en Angola ${ }^{4}$ [traducción propia] (MESCTI, 2019a: 8).

Es por esta razón, que se considera fundamental la creación de plataformas de cooperación científica y tecnológica entre los actores de las distintas IES angolanas, a través de procesos de selección de especialistas con resultados relevantes en sus áreas de formación, en aras de establecer parcerías estratégicas con investigadores internacionales, que contribuyan a perfeccionar el proceso de producción y difusión científica a una escala global. En este sentido, una de las formas de cooperación está relacionada con la participación en el desarrollo de revistas científicas, a través de la consolidación de consejos editoriales, grupos de árbitros, entre otros, que contribuyan al desarrollo de procesos eficientes al respecto.

De forma complementaria Guambe; Bueno-de-la-Fuente (2013); Gomes de Oliveira Reis; De Melo Catelão (2017) enfatizan que para que el desarrollo y la sistematización del proceso de investigación científica de una institución esté consolidado, es necesario, su registro en algún suporte físico o electrónico, destacando las revistas científicas como una de las formas más usadas para la difusión de los resultados, a una mayor escala nacional e internacional.

Sin embargo, es un proceso de aprendizaje también, ya que, en el contexto angolano, los docentes e investigadores no presentan profundos conocimientos sobre los procedimientos de una revista científica y su trayectoria, desde la recepción de propuestas hasta su publicación. Esto significa que, a pesar de que las revistas científicas son medios por excelencia de difusión de resultados científicos, esta comprensión debe ser fortalecida por un proceso de capacitación intencional, para desarrollar la cultura de circulación de información científica, técnica, pedagógica y administrativa (Gungula; Castillero; Barbosa Rodrigues, 2017; Gomes de Oliveira Reis; De Melo Catelão, 2017).

Se puede observar entonces, que contextualizando la realidad en investigación en Angola, existe mucho por hacer al respecto, y es significativo, el interés por parte de las IES y del Estado de mejorar los procesos; sin embargo, se necesita tiempo y dedicación pues apenas se inicia en destacar la importancia de la producción científica como forma de desarrollo nacional, por lo que, cualquier iniciativa que se lleve a cabo en este sentido será favorable, en la búsqueda de las

4 A precariedade de muitas instalações, a escassez de equipamentos de laboratório, a turbo-docência, a qualidade questionável do ensino-aprendizagem reforçada pela escassa relação entre a teoria e aprática, a incipiência da investigação científica e a existência de uma cultura do plágio são factores críticos que limitam o desenvolvimento do Subsistema do Ensino Superior, da investigação científica e da transferência de tecnologia e da inovação em Angola. 
formas a través de las cuales los docentes e investigadores encuentren el mejor camino para el aprendizaje y mejoramiento profesional.

\section{Alternativas para el avance de la investigación científica en el contexto angolano: la creación de revistas como una de ellas}

Hoy, el perfeccionamiento del proceso de la investigación científica en el contexto angolano, constituye el centro de debates realizados en distintas IES y centros de desarrollo tecnológico, debido a la necesidad de demostrar su rol en la solución de problemas sociales, analizar la política y estrategia nacional de desarrollo científico, evaluar los procesos de elaboración de planes de desarrollo institucional, así como, comprender las principales causas de la baja producción y publicación científica en medios creíbles (MESCTI, 2019b; Artigas; Gungula, 2020).

En Angola, estos procesos de investigación científica y de desarrollo tecnológico, son desarrollados, fundamentalmente en las universidades públicas y privadas, así como, en determinados ministerios, definidos por el Estado. Entre ellos se destacan: Ministerio de la Agricultura, Ministerio de la Cultura, Ministerio de Pescas, Ministerio de Geología y Minas, Ministerio de Urbanismo y Habitación, Ministerio de Obras Públicas, Ministerio de Ciencia y Tecnología, Ministerio de Defensa, Ministerio de Salud, Ministerio de Industria, Ministerio de Educación, Ministerio de Telecomunicaciones y Tecnologías de Información, Ministerio del Comercio y el Estado Mayor General de las Fuerzas Armadas (Ministério da Ciência e Tecnologia, $\mathrm{s} / \mathrm{f})$.

Dentro de la estructura orgánica de los referidos ministerios, se encuentran distintos institutos, escuelas superiores politécnicas, facultades, laboratorios, museos, archivos, servicios, direcciones nacionales, centros de investigación y de desarrollo tecnológico (Ministério da Ciência e Tecnologia, s/f; PLANCTI, 2014-2015).

Analizando la limitada visibilidad nacional e internacional de la producción científica realizada por dichas instituciones, fácilmente se concluye que, el perfeccionamiento de los procesos de investigación científica en correspondencia con las estrategias de desarrollo sustentable de Angola, debe constituirse en necesidad estratégica (Artigas; Gungula, 2020). Por ello, la primera alternativa consiste en la adaptación a los cambios científicos y tecnológicos globales, para que las distintas IES se transformen en verdaderos centros por excelencia de sistematización y de intercambio de conocimientos, a través de parcerías estratégicas con las IES angolanas e internacionales.

En este sentido, se debe igualmente, mejorar la articulación de los procesos sustantivos, reestructurar la concepción de desarrollo institucional, donde los docentes, los investigadores y demás implicados en el proceso asuman su rol para la materialización eficiente de los programas de capacitación especializada, de formación graduada y postgraduada en la modalidad presencial y a distancia.

Según el MESCTI (2019a), entre las principales causas de la baja productividad científica en Angola se destacan: la falta de financiamiento y movilización de 
fondos para la ciencia; escasez de recursos humanos diferenciados para la investigación científica; deficiente organización de las instituciones de investigación y desarrollo; así como, la falta de evaluación interna y externa de las IES.

Así, se torna necesario encaminar la segunda alternativa, hacia los procesos de gestión universitaria, capacitación del personal docente y administrativo, elaboración y gestión de proyectos multidisciplinares, en aras de desarrollar nuevas habilidades y competencias por parte de los actores que dinamizan las distintas IES y centros de desarrollo e investigación tecnológica.

Además de las limitaciones descritas, las alternativas propuestas deben reforzar la capacidad de organización y cooperación interinstitucional, creando condiciones que estimulen los docentes e investigadores con resultados relevantes, a través de su participación en eventos científicos nacionales e internacionales, así como, la realización de cursos de maestrías, doctorados y postdoctorados, entre otros beneficios y reconocimientos (Gungula; Faustino; Torrecilla Díaz, 2015).

Para ello, como tercera alternativa se torna fundamental estimular y fortalecer las iniciativas de creación y gestión de revistas científicas, como medios creíbles de difusión del conocimiento generado por la comunidad académica nacional e internacional, teniendo en cuenta que en estos procesos, se requiere el cumplimiento de una serie de requisitos que, al lograrse, los investigadores desarrollan habilidades y competencias investigativas que contribuyen al perfeccionamiento de la producción científica y su visibilidad a una escala global.

Este proceso, requiere cada vez más la comprensión y la participación activa/continua de los docentes e investigadores en actividades editoriales científicas, en comisiones de eventos científicos nacionales e internacionales, en procesos de evaluación de programas y proyectos de desarrollo local, así como, su registro en redes nacionales e internacionales de circulación de información científica, como la cuarta alternativa.

La quinta alternativa consiste en la capacitación para la diferenciación consciente de las distintas formas de redacción de resultados científicos, tales como: monografías, disertación, tesis, ponencias, artículos científicos, trabajos de progresión en la carrera docente, informes de proyectos de investigación científica, entre otros, debido a sus características específicas y genéricas, considerando las normas de cada trabajo, institución, así como las costumbres de cada país.

Los trabajos mencionados, deben cumplir en su generalidad criterios como: clasificación del trabajo, estructura, extensión, objetividad, precisión, rigor metodológico y los fines que se persiguen con la publicación de estos. En este contexto, es fundamental que se perciba la perspectiva de cada investigador, buscando dar a conocer sus resultados a la comunidad científica nacional e internacional, o procurando presentar un informe ante la institución o empresa que lo ha solicitado.

Del mismo modo, es esencial que se comprenda la diferenciación del propósito de quienes realizan investigaciones y presentan resultados para obtener algún grado académico, de los que se dedican al desarrollo científico de sus especialidades, para 
que se determine con precisión, el tipo de trabajo a realizar en cada ocasión y contexto (Sabino, 1992).

Así, urge mejorar los procedimientos de reestructuración de la forma como se interrelacionan las universidades, las empresas y la sociedad, en busca del desarrollo sustentable de Angola, a través del desarrollo de una cultura de excelencia, calidad, innovación, emprendimiento, eficiencia y de evaluación del desempeño profesional e institucional (Constituição da República de Angola, Assembleia Nacional, 2010).

Se puede observar entonces como la creación de revistas científicas se constituye en la alternativa que globaliza el resto de las alternativas para el mejoramiento de la investigación científica, de manera que las IES puedan a través de las mismas contribuir a la capacitación y al mismo tiempo el aumento de la visibilidad de la productividad de los investigadores, contribuyendo al conocimiento de la diferenciación de las distintas formas de difusión del conocimiento, la socialización a través de eventos científicos relacionados y la construcción de redes de conocimiento entre investigadores nacionales e internacionales, al compartir actividades científicas inherentes a consejos editoriales y revisiones por pares fundamentalmente.

\section{Métodos}

Buscando bases que confirmen la pertinencia de la problemática tratada como resultado de una investigación de naturaleza documental, se revisó lo escrito hasta el momento sobre productividad y revistas científicas en Angola para contextualizar la situación actual.

También se realizó una revisión en el portal ISSN internacional con respecto a revistas científicas del país, hasta diciembre del 2019, pudiendo identificar diez revistas científicas electrónicas asociadas a IES angolanas, activas y arbitradas, como se mencionan a continuación:

a) Sapientiae: Revista de Ciências Sociais, Humanas e Engenharia (http://publicacoes.uor.ed.ao/index.php/sapientiae);

b) RAS: Revista Angolana de Sociologia (https://journals.openedition.org/ras/);

c) Mulemba: Revista Angolana de Ciências Sociais (https://journals. openedition.org/mulemba/);

d) ROP: Órbita Pedagógica (http://revista.isced-hbo.ed.ao/rop/index.php/ROP);

e) RAC: Revista Angolana de Ciências (http://publicacoes.scientia.co.ao/ojs2/ index.php/rac);

f) Revista Sol Nascente (http://www.ispsn.org/revista-sol-nascente);

g) Revista Angolana de Extensão Universitária (http://www.portalpensador .com/index.php/RAEU-BENGO/index);

h) Tundavala: Revista Angolana de Ciências (http://www.portalpensador.com/ index.php/Tdvla);

i) Kulonguesa: Tecnologia - Educação - Sutentabilidade (https://kulongesa. ispls.ao/index.php/kulongesa-tes). 
j) Revista angolana de ciências e tecnologias de informação e comunicação (http://racitic.isutic.gov.ao/index.php/racitic/issue/archive)

Una de ellas, no está disponible en la web, Revista Angolana de Ciência e Tecnologias de Informação e Comunicação (http://racitic.isutic.gov.ao/index.php/ racitic/issue/archive).

De esta manera, las nueve revistas encontradas se revisaron en su enlace web, mostrando:

a) Instituciones a las que pertenecen (públicas o privadas).

b) Último volumen/número publicado.

c) Índices y bases de datos en las que se encuentran.

d) Distribución nacional e internacional de los miembros del consejo editorial de las revistas.

El análisis se muestra a través de tablas en los resultados a continuación, de manera que pueda ser analizada de forma más amigable.

\section{Resultados y discusión}

A continuación, se presenta la información encontrada en los enlaces web de las revistas mencionadas, así mismo, luego de cada tabla se hace un análisis de la información encontrada seguida de una discusión contrastado con resultados previos en estudios similares.

a) Instituciones a las que pertenecen las revistas científicas. Tabla 1

\begin{tabular}{|l|r|r|l|}
\hline \multirow{2}{*}{ Revista Científica } & \multicolumn{2}{|c|}{ Tipo de institución } & Nombre de la institución \\
\cline { 2 - 5 } Publica & Privada & \\
\hline $\begin{array}{l}\text { Sapientiae: Revista de } \\
\text { Ciências Sociais, Humanas e } \\
\text { Engenharia }\end{array}$ & X & $\begin{array}{l}\text { Universidade Óscar Ribas } \\
\text { (UÓR) }\end{array}$ \\
\hline $\begin{array}{l}\text { RAS: Revista Angolana de } \\
\text { Sociologia }\end{array}$ & $\mathrm{X}$ & $\begin{array}{l}\text { Sociedade Angolana de } \\
\text { Sociologia (SASO). }\end{array}$ \\
\hline $\begin{array}{l}\text { Mulemba: Revista Angolana } \\
\text { de Ciências Sociais }\end{array}$ & $\begin{array}{l}\text { Faculdade de Ciências Sociais } \\
\text { da Universidade Agostinho } \\
\text { Neto (FCS-UAN). }\end{array}$ \\
\hline ROP: Órbita Pedagógica & & $\begin{array}{l}\text { Instituto Superior de Ciências } \\
\text { de Educação do Huambo } \\
\text { (ISCED). }\end{array}$ \\
\hline $\begin{array}{l}\text { RAC: Revista Angolana de } \\
\text { Ciências }\end{array}$ & X & $\begin{array}{l}\text { Associação Multidisciplinar de } \\
\text { Investigação Científica } \\
\text { (AMIC). }\end{array}$ \\
\hline Revista Sol Nascente & X & $\begin{array}{l}\text { Instituto Superior Politécnico } \\
\text { Sol Nascente (ISPSN). }\end{array}$ \\
\hline
\end{tabular}




\begin{tabular}{|l|r|r|l|}
\hline $\begin{array}{l}\text { Revista Angolana de Extensão } \\
\text { Universitária }\end{array}$ & X & $\begin{array}{l}\text { Escola Superior Pedagógica do } \\
\text { Bengo (ESPB). }\end{array}$ \\
\hline $\begin{array}{l}\text { Tundavala: Revista Angolana } \\
\text { de Ciências }\end{array}$ & & X & $\begin{array}{l}\text { Instituto Superior Politécnico } \\
\text { Tundavala } \\
\text { (ISPTUNDAVALA). }\end{array}$ \\
\hline $\begin{array}{l}\text { Kulonguesa: Tecnologia - } \\
\text { Educação - Sutentabilidade }\end{array}$ & X & $\begin{array}{l}\text { Escola Superior Politécnica da } \\
\text { Lunda Sul (ESPLS). }\end{array}$ \\
\hline
\end{tabular}

Fuente: elaboración propia.

Como puede observarse el 55,56\% de las revistas científicas son desarrolladas en instituciones privadas, mientras que el $44,44 \%$ en instituciones públicas. Pereira; Casanova; Pire (2014) señalaban que en Venezuela la mayor cantidad de revistas científicas se encontraban en instituciones públicas, debido a que las mismas se encuentran enfocadas a la investigación, mientras que las privadas se enfocan a la docencia; se puede observar en Angola un fenómeno contrario, se visualiza el interés de las instituciones privadas por diferenciarse a través de los procesos de investigación.

La diferenciación entre el tipo de institución que edita la revista científica es importante debido a que mediante esta diferenciación se observa la intención o interés de los entes públicos en cuanto al financiamiento de estos procesos. Teniendo en cuenta la importancia de las revistas científicas dentro de los indicadores de productividad institucional y de los investigadores científicos, esto muestra como en las instituciones públicas no han logrado diferenciarse en cuanto a la creación de revistas para llevar la delantera con relación a las instituciones privadas.

b) Ultimo volumen/número publicado en las revistas científicas angolanas. Tabla 2

\begin{tabular}{|l|l|}
\hline Revista Científica & Último Volumen/Numero \\
\hline $\begin{array}{l}\text { Sapientiae: Revista de Ciências Sociais, } \\
\text { Humanas e Engenharia }\end{array}$ & $5-2$ (Enero-Junio 2020) \\
\hline RAS: Revista Angolana de Sociologia & 14 (2014) \\
\hline $\begin{array}{l}\text { Mulemba: Revista Angolana de Ciências } \\
\text { Sociais }\end{array}$ & $6-12$ (2016) \\
\hline RÓP: Órbita Pedagógica & $7-1$ (Enero-Abril 2020) \\
\hline RAC: Revista Angolana de Ciências & $1-2$ (Julio-Diciembre 2019) \\
\hline Revista Sol Nascente Angolana de Extensão & 17 (Abril 2020) \\
\hline $\begin{array}{l}\text { Revista (2019) resultados de conferencia } \\
\text { Universitária }\end{array}$ & $2-1(2015)$ \\
\hline Tundavala: Revista Angolana de Ciências & $2-1(2020)$ \\
\hline $\begin{array}{l}\text { Kulonguesa: Tecnologia - Educação - } \\
\text { Sutentabilidade }\end{array}$ & \\
\hline
\end{tabular}

Fuente: elaboración propia.

Revisando la tabla 2 (estos resultados corresponden a los encontrados hasta abril del 2020), se observa que el 44,44\% de las revistas científicas se encuentran actualizadas con los números del año en curso, el restante se encuentra con 
números de años anteriores. Se observa entonces como existe el impulso en la creación de estas, pero su continuidad es el proceso más difícil. Se excluye en adelante la Revista Angolana de Extensão Universitária pues al parecer solo se usó para un evento científico, por tanto, no puede ser tomada como referencia sobre los siguientes elementos. Como señalan Abadal; Rius (2006) y coincide Paz Enrique (2018), uno de los aspectos formales que debe cumplir una revista científica tiene que ver con la periodicidad. También Díaz-Pérez; Hernández-Sánchez; NúñezGarduño (2018: 20) señalan que "uno de los factores fundamentales es el cumplimiento de la periodicidad; esto es, cuando la revista es publicada al principio del periodo, los textos tienen mayor tiempo de vigencia para ser consultados”.

Lo que quiere decir, que la publicación a tiempo y especialmente a inicio del periodo que la revista científica enuncia da una mayor posibilidad a que el conocimiento se cite de forma rápida y tenga más vigencia, en este caso, existen revistas científicas angolanas que desde el año 2014, 2015 y 2016 no han publicado nuevos números, mostrando un atraso de hasta seis (6) años en el proceso. Estas revistas científicas para poder continuar deberán ponerse al día con todos los números atrasados, lo que implica un gran trabajo por parte del personal involucrado.

Suárez Guava (2017) reseña algunos de los hechos acontecidos en Colombia con el tema de las revistas científicas y ciertamente hace referencia a que muchos de los proyectos desfallecen por falta de reconocimiento, las revistas científicas conllevan mucho trabajo que muchas veces es poco recompensado. En el proceso de creación de revistas científicas y su ubicación en índices de alto impacto pasa un tiempo en el que la inversión parece no tener retorno, por tanto, muchas revistas científicas son abandonadas antes de poder cumplir los requisitos de calidad, pero sobre todo en antigüedad/reconocimiento para poder ubicarse en los índices internacionales.

Se debe tener en cuenta que para que la revista científica sea reconocida y los investigadores la tomen como referencia para citar sus trabajos, debe tener cierta antigüedad en el mercado, pero sobre todo, debe publicar trabajos que los investigadores consideren de referencia en el área de conocimiento, lo cual, se logra cuando investigadores reconocidos publican en la misma, y así, aquellos que revisen sobre dichos autores ubicarán la revista como punto de referencia, generando al mismo tiempo interés por publicar en la misma; es un proceso un poco complicado y en cierta forma entrampado para revistas nuevas, pero puede lograrse.

c) Índices y bases de datos en los que se encuentran registradas las revistas científicas angolanas. Tabla 3

\begin{tabular}{|l|l|}
\hline Revista Científica & Índices/Bases de datos \\
\hline Sapientiae: Revista de Ciências Sociais, & $\begin{array}{l}\text { Actualidad Iberoamericana, I2OR, } \\
\text { Humanas e Engenharia }\end{array}$ \\
& Dailnet, REDIB, Google Scholar, \\
& MIAR, OAJI, DOAJ, CiteFactor, SIS, \\
\hline
\end{tabular}




\begin{tabular}{|c|c|}
\hline & $\begin{array}{l}\text { Deycrit Sur, Science Library Index, } \\
\text { Livre, ERIHPLUS, LatinREV, ROAD } \\
\text { (ISSN), Sherpa Romeo, Journals for } \\
\text { free, Core, WorldCat, Base, Redalyc, } \\
\text { Crossref (DOI). }\end{array}$ \\
\hline RAS: Revista Angolana de Sociologia & Open Edition Journal. \\
\hline $\begin{array}{l}\text { Mulemba: Revista } \\
\text { Ciências Sociais }\end{array}$ & Open Edition Journal. \\
\hline RÓP: Órbita Pedagógica & $\begin{array}{l}\text { LatinREV, EuroPub, } \\
\text { ERIHPLUS, REDIB, } \\
\text { Iberoamericana, CiteFactor, BAStualidad } \\
\text { Livre, Sumarios, ROAD (ISSN), } \\
\text { Elektronische, ReasearchBib, OAJ, } \\
\text { TIB, WorldCat, DRJI, I2OR, Sherpa } \\
\text { Romeo, Google Scholar, Rederca. }\end{array}$ \\
\hline RAC: Revista Angolana de Ciências & $\begin{array}{l}\text { EuroPub, ROAD (ISSN), LatinREV, } \\
\text { Google Scholar, BASE, TIB, WorldCat, } \\
\text { SIS, Actualidad Iberoamericana, } \\
\text { LEIPZIG, Deycrit Sur, Sumarios, DRJI, } \\
\text { CiteFactor, } \\
\begin{array}{l}\text { ReasearchBib, Livre. }\end{array}\end{array}$ \\
\hline Revista Sol Nascente & No muestra índices/bases de datos. \\
\hline $\begin{array}{llll}\text { Tundavala: } & \text { Revista } & \text { Angolana } & \text { de } \\
\text { Ciências } & & & \\
\end{array}$ & No muestra índices/bases de datos. \\
\hline $\begin{array}{l}\text { Kulonguesa: Tecnologia - Educação - } \\
\text { Sutentabilidade }\end{array}$ & Google $S$ \\
\hline
\end{tabular}

Fuente: elaboración propia.

Se reconoce la diferencia entre índices y bases de datos, que recae principalmente en la capacidad de medir con respecto a ciertos elementos el posicionamiento de las revistas científicas, de manera que la tendencia es hacia la presentación de rankings al respecto de ciertos criterios de calidad, sin embargo, aunque la tendencia es hacia la primacía de los índices sobre las bases de datos o repositorios, se presentarán aquí sin discriminación, tomando en cuenta que las revistas científicas en el contexto angolano aún no se ubican en ningún ranking internacional de los más importantes para hacer referencia.

Como puede observarse solo el 37,5\% (3/8) de las revistas científicas angolanas se encuentran en más de 3 (tres) índices/bases de datos, algunas incluso solo aparecen en uno o en ninguno, lo que hace relacionarlo con la actualización de éstas, pues es uno de los requisitos fundamentales para la inclusión en algunos de ellos.

Analizando los índices/bases de datos en que las referidas revistas científicas angolanas se encuentran indexadas, fácilmente se concluye que se requiere de capacitación especializadas para su perfeccionamiento. En este contexto, solo dos revistas se encuentran indexadas en el DOAJ (Sapientiae y Revista Órbita 
Pedagógica) aun teniendo en cuenta que todas son de acceso abierto, una en la Redalyc (Sapientiae) y ninguna en las más prestigiosas bases de datos internacionales como: Web of Science, Scopus, entre otras. Hay que tomar en cuenta que la indización es fundamental para el logro de posicionamiento, y que los investigadores se interesen en publicar. Otro elemento importante para señalar es la presencia de una de las revistas científicas $(11,11 \%)$ en CrossRef, denotando que solo una de ellas posee el DOI (Identificador de Objeto Digital), elemento de suma importancia en la actualidad para generar un seguimiento completo de los documentos en la web, generar registro completo de sus citaciones, y, por tanto, contribuir a la difusión y registro del conocimiento generado en la web.

Es importante resaltar que, en los países desarrollados las carreras académicas están ligadas al logro de la difusión de la ciencia a través de instrumentos de alto impacto, esto quiere decir, de revistas científicas que se encuentren en las mejores posiciones en cuanto a índices de citación y referencia. Artigas; Casanova (2020) hacen referencia a como las instituciones prácticamente obligan a sus integrantes para publicar en revistas científicas de alto factor de impacto, pues esto contribuirá al posicionamiento de la institución en ranking internacionales y, por tanto, a obtener mayor apoyo de los entes gubernamentales. En este sentido, es lógico que los investigadores en el ámbito internacional busquen para publicar revistas científicas que se encuentren en los mejores índices, lo que perjudica a las revistas científicas nuevas, que deben hacer un esfuerzo mayor por elevar su posición.

Este es el caso de Angola, pues existen investigadores reconocidos en el ámbito nacional, pero seguramente preferirán publicar en revistas científicas internacionales que incrementen sus índices de citación y referencia. Haciendo que las revistas científicas nacionales tengan más dificultades para lograr el número de artículos requeridos para cada edición, disminuyendo la posibilidad de ser incluida en los índices internacionales más rigurosos/reconocidos.

Sin embargo, el esfuerzo no desmaya, no puede dejarse de lado el gran avance de la revista SAPIENTIAE que logró en el año 2019 ser incluida en Redalyc como la primera revista científica africana en ingresar al índice, lo que ha traído consigo incluso posibilidades de proyectos conjuntos y de incrementar la visibilidad de la ciencia angolana para Latinoamérica, España, Portugal y el Caribe. Redalyc maneja un portal denominado Marcalyc en el cual se marcan los documentos y pueden extraerse los formatos PDF, HTML y EPUB que pueden servir de soporte a las revistas científicas para reducir sus costos de montaje de los documentos, aminorando así el trabajo de los equipos de edición y sirviendo de soporte para la difusión de la ciencia en el mundo.

Cada avance en el logro de índices significa más posibilidad de ser leídos y citados internacionalmente, lo cual, a largo plazo significa una mejora en el posicionamiento. Por tanto, cada vez que una revista científica se incluye en un índice amplia las posibilidades de esta de ser reconocida en el ámbito internacional, al mismo tiempo, que da la posibilidad de reconocimiento del país en el cual se encuentra registrada y de seguir sumando en sus indicadores de productividad. 
d) Distribución nacional de los miembros del consejo editorial de las revistas científicas angolanas. Tabla 4

\begin{tabular}{|l|r|r|r|}
\hline Revista Científica & \multicolumn{1}{l|}{ Total } & Nacional & \% nacionales \\
\hline $\begin{array}{l}\text { Sapientiae: Revista de Ciências Sociais, } \\
\text { Humanas e Engenharia }\end{array}$ & 31 & 10 & $32,26 \%$ \\
\hline RAS: Revista Angolana de Sociologia & 32 & 13 & $40,63 \%$ \\
\hline $\begin{array}{l}\text { Mulemba: Revista Angolana de Ciências } \\
\text { Sociais }\end{array}$ & 56 & 37 & $66,07 \%$ \\
\hline RÓP: Órbita Pedagógica & 37 & 20 & $54,05 \%$ \\
\hline RAC: Revista Angolana de Ciências & 24 & 19 & $79,17 \%$ \\
\hline Revista Sol Nascente & 42 & 14 & $33,33 \%$ \\
\hline Tundavala: Revista Angolana de Ciências & 6 & 4 & $66,66 \%$ \\
\hline $\begin{array}{l}\text { Kulonguesa: Tecnologia - Educação - } \\
\text { Sutentabilidade }\end{array}$ & 23 & 14 & $60,87 \%$ \\
\hline
\end{tabular}

Fuente: elaboración propia.

Como se puede ver en la tabla 4, todas las revistas científicas angolanas tienen participación nacional en sus equipos editoriales, se debe tener en cuenta que posiblemente la revista Tundavala no tenga la información correctamente, ya que presenta muy pocos miembros. Muchas veces las revistas científicas no completan toda la información requerida en sus sitios web, siendo esta una de las razones por las cuales no son aceptadas en los índices internacionales, ya que la única manera de obtener información es a través del sitio web en el cual se encuentran, básicamente, es la fuente de información más confiable para autores y cualquier interesado en conocer los procesos de la revista.

Aunque los índices solicitan que la participación nacional no exceda el $40 \%$ en la mayoría de los casos, la idea de mostrar esta información tiene que ver con la muestra de que sí existen investigadores nacionales involucrados en la edición de las revistas científicas y en algunos casos incluso se repiten investigadores entre las revistas en función de su conocimiento al respecto. Lo cual deja ver que es posible seguir avanzando en el tema en función de lo que se ha logrado a la fecha.

Por citar algún índice, Redalyc (Red de Revistas Científicas de América Latina y el Caribe, España y Portugal) exige como un Criterio Altamente Valorado (CAV) que al menos el $75 \%$ de los integrantes del consejo editorial deben ser ajenos a la entidad editora (Redalyc, 2020). Esto quiere decir, que solo el 25\% de los miembros deben ser de la misma institución a la que pertenece la revista, dejando libertad en cuanto a la división de los participantes externos (de otra institución e internacionales).

Es importante resaltar que tomando en cuenta estos datos, se puede afirmar que sí existen investigadores en el ámbito nacional que han participado en procesos de creación y edición de revistas científicas, los cuales pueden convocarse para crear proyectos similares con otras instituciones y apoyar en el desarrollo de entrenamientos a los profesores e investigadores interesados en la mejoría de su labor profesional, contando así, con capital humano altamente calificado en el ámbito nacional que pueden servir de apoyo a las instituciones que deseen iniciarse 
en el camino de la edición de revistas científicas como alternativa de mejoramiento del proceso investigativo.

\section{Conclusiones}

Las tendencias científicas globales apuntan a las revistas científicas electrónicas como el medio por excelencia y más rápido de difusión del conocimiento científico, un indicador que debe ser privilegiado en el contexto angolano de educación superior, ciencia, tecnología e innovación. En este sentido, en este artículo se examinaron las revistas científicas angolanas que se encuentran registradas en el portal ISSN internacional y su situación actual como alternativa de mejoramiento del proceso investigativo de las distintas instituciones, llegando a las siguientes conclusiones:

- Las revistas científicas registradas ante el portal ISSN internacional pertenecientes a Angola son 10 (diez), sin embargo, solo 9 (nueve) de ellas pudieron ser ubicadas en los enlaces web de referencia en dicho portal.

- Una de ellas se utilizó solo para la publicación de unas memorias de un evento, por tanto, se excluyó a mitad de la evaluación, esto es, la Revista Angolana de Extensão Universitária.

- De las 9 (nueve) revistas científicas evaluadas en el estudio, un poco más de la mitad pertenecen a IES privadas, logrando que no exista diferencia significativa en cuanto al interés entre las IES públicas y privadas.

- Menos de la mitad de estas se encuentran actualizadas y las que se encuentran actualizadas se encuentran en su mayoría en más de 3 (tres) índices/bases de datos, los cuales no son los de mayor impacto internacional, sin embargo, contribuyen a que las revistas científicas angolanas vayan tomando posición para su posibilidad futura de solicitar los índices de mayor impacto.

- Se observa la participación de investigadores nacionales en los consejos editoriales de las referidas revistas, lo que señala el interés que ha tomado la difusión científica en los últimos años y como los investigadores se han integrado a los procesos editoriales como parte de sus labores académicas.

Los docentes universitarios e investigadores científicos angolanos deben comprender que esta alternativa de mejoramiento influye significativamente en la investigación científica y la forma como la misma es abordada, ya que, en el proceso de revisión y mejoramiento de los procesos editoriales se aprende a manejar la difusión de la ciencia internacionalmente, lo cual sirve para el mejoramiento de los procesos internos de investigación, desde lo individual a lo colectivo.

El desarrollo de Angola debe centrar sus bases en la articulación de los procesos sustantivos de la universidad - la educación, la investigación científica, la extensión universitaria y la internacionalización - como procesos indisolubles. 
El limitado número de revistas científicas arbitradas, indexadas y editadas por las distintas IES públicas y privadas angolanas, es un indicador para que el Estado dedique particular atención a estos procesos, como expresión de su interés en posicionar al menos 2 (dos) IES en los rankings académicos internacionales, y estrategia para aumentar la visibilidad de su compromiso con el desarrollo de la ciencia, la tecnología y la innovación.

Las publicaciones científicas en revistas arbitradas e indexadas constituyen un resultado relevante que aporta valor y avales de calidad a los procesos de investigación en la mayoría de las áreas del conocimiento y se constituyen en la forma fundamental de comunicación, divulgación y evaluación en correspondencia con las estrategias de cada país, siendo además, fuentes de debate científico y base referencial indispensable para otros investigadores.

Teniendo en cuenta que en el contexto internacional, todo docente o investigador, durante el proceso de formación avanzada (doctorados y postdoctorados), está obligado a cumplir el requisito de publicación de los resultados esenciales de su investigación en un mínimo de 2 (dos) a 3 (tres) publicaciones indexadas en los sistemas de indización y resumen regionales e internacionales tales como: catálogos, directorios, índices de citas, bases de datos, libros arbitrados e indexados, entre otros medios creíbles de publicación de resultados científicos.

En este sentido, se observa como a través del desarrollo de una de las revistas científicas angolanas en los últimos años (SAPIENTIAE) se configura como viable y posible el desarrollo de otras revistas científicas, que pueden constituirse como alternativa para la publicación de resultados de investigaciones científicas realizadas durante las referidas formaciones de postgrado, según lo establecido en los respectivos programas de formación como un criterio/requisito internacional utilizado en la mayoría de los países americanos, europeos, africanos y asiáticos.

Así, en el contexto angolano de educación superior, ciencia, tecnología e innovación, urge normar las exigencias para el cumplimiento de este requisito, teniendo en cuenta la necesidad de elevar la calidad de las publicaciones de los resultados de las investigaciones científicas en revistas de prestigio nacional e internacional, así como establecer las condiciones para su cumplimiento por parte de las IES autorizadas para la formación avanzada en determinada especialidad.

Por último, la difusión de la ciencia en Angola a través de revistas científicas, constituye una oportunidad de cooperación estratégica entre IES nacionales e internacionales, ya que, como en las revistas científicas debe publicarse por lo menos un $60 \%$ de contenido externo a la IES editora, se pueden abrir espacios de intercambio de procedimientos de desarrollo entre las mismas, y así, impulsar la publicación en las otras revistas de instituciones nacionales creando redes de cooperación y revisión por pares.

\section{Referencias bibliográficas}


Abadal, E.; Rius, L. (2006). Revistas Cientificas digitales: características e indicadores. Revista de Universidad y Sociedad del Conocimiento. 3 (1), 6-20. http://dx.doi.org/10.7238/rusc.v3i1.272

André, A. M. Ch. (2016). Avaliação da qualidade em instituições de ensino superior públicas angolanas. (Tesis de doctorado). Universidad de Aveiro, Dept. de Educación y Psicologia.

Artigas, W.; Casanova, I. (2020). Influencia de las redes sociales académicas en la construcción de la identidad digital latinoamericana. Anales de Documentación, 2020, 23 (2). http://dx.doi.org/10.6018/analesdoc.397551

Artigas, W.; Gungula, E. W. (2020). Gestión de revistas a través de OJS: Experiencia de éxito Angolana. e-Ciencias de la Información, 10 (1), 3-18. https://doi.org/10.15517/eci.v10i1. 39771

Assembleia Nacional (2010). Constituição da República de Angola. Disponible en https://www.wipo.int/edocs/lexdocs/laws/pt/ao/ao001pt.pdf

Calderón, A.; Marshal França, C. (2018). Rankings acadêmicos na educação superior: tendências da literatura ibero-americana. Avaliação: Revista da Avaliação da Educação Superior, 23 (02), 448-466. http://dx.doi.org/10.1590/s1414-40772018000200010

Chirino-Ramos, M. V. (2012). Didáctica de la formación inicial investigativa en las universidades de ciencias pedagógicas. Varona, (55), 18-24. Disponible en: https://www.redalyc.org/pdf/3606/Resumenes/Resumen_360633907004_1.pdf

Díaz-Pérez, G.; Hernández-Sánchez, V.; Núñez-Garduño, I. (2018). Convergencia Revista de Ciencias Sociales y los avatares hacia su digitalización. En Revistas científicas mexicanas. Retos de calidad y visibilidad en acceso abierto. Coordinadores Jan Rus, Astrid Maribel Pinto Durán. Chiapas, Universidad de Ciencias y Artes de Chiapas.

Didod Aupetit, S. (2014). La UNESCO y la educación superior, 2014

e2017: Aportes d Reunión de Cátedras UNESCO sobre la educación superior, las TIC en la educación y los profesores. Disponible en http://www.unesco.org/new/fileadmin/MULTIMEDIA/ HQ/ED/pdf/UNESCO-summary-report-chairs-2014-1.pdf

Estratégia Nacional de Ciência, Tencologia e Inovação - ENCTI, (2011). Decreto Presidencial n. ${ }^{\circ}$ 201/11. Angola.

Faustino, A.; Gungula, E. W.; Craib, E. (2015). El enfoque dialéctico en la formación matemática y su repercusión científico-tecnológica en el contexto social. Prisma Social. (14), 574-602.

Faustino, A.; Pérez Sánchez, N.; Gungula, E. W. (2017). Formación del Pensamiento Complejo Ingenieril en la Universidad “Óscar Ribas" Telos. Revista de Estudios Interdisciplinarios en Ciencias Sociales, 19 (3), 523-544.

Fuentes, H. C. G., Montoya, J. R., \& Fuentes, L. S. (2011). La formación en la Educación Superior. Desde lo Holístico, Complejo y Dialéctico de la Construcción del Conocimiento Científico. Universidad de Oriente, Centro de Estudio de Educción Superior "Manuel F. Gran”. Ediciones UO.

Gomes de Oliveira Reis, S.; De Melo Catelão, E. (2017). Criação de um periódico científico online em uma instituição de ensino superior pública. Informação \& Informação, 22, (2), pp. 546 - $563 . \quad$ http://dx.doi.org/10.5433/19818920.2017v22n2p546

Guambe, M. F.; Bueno-de-la-Fuente, G. (2013). Disponibilidade em Acesso Aberto da produção científica dos países da África Lusófona. Revista de Ciência da Informação e Documentação, 4 (2), 5-19. https://doi.org/10.11606/issn.2178-2075.v4i2p5-19

Gungula, E. W.; Castillero, J.; Barbosa Rodrigues, M. (2017). Visibilidade dos Sistemas de Créditos Académicos nos Países Lusófonos. Telos. Revista de Estudios Interdisciplinarios en Ciencias Sociales. 19 (2), 288-307. 
Gungula, E. W.; Dieguez, R.; Pérez Ugartemendía, E. (2015). Estrategia didáctica para el perfeccionamiento del proceso de formación interpretativa en la matemática superior. Revista Actualidades Investigativas en Educación, 15 (2), 1-41. http://dx.doi.org/10.15517/aie.v15i2.18954

Gungula, E. W.; Faustino, A. (2018). Dilema da formação matemática em Angola: ¿falta de iniciativas próprias ou de compromisso com a qualidade de ensino? Revista $\begin{array}{llllll}\text { Actualidades Investigativas en } & \text { Educación. } & \text { (3), } 22 .\end{array}$ http://dx.doi.org/10.15517/aie.v18i3.33459

Gungula, E. W.; Faustino, A.; Pérez Ugartemendía, E. (2013). El contexto angolano de formación matemática: Un problema que se arrastra desde la base. Avaliação: Revista da Avaliação da Educação Superior, 18 (2), 487-499. http://dx.doi.org/10.1590/S141440772013000200013

Gungula, E. W.; Faustino, A.; Torrecilla Díaz, R. (2015). Challenges and Prospects for the Improvement of the Angolan Mathematical Training. Education, 5(2), 55-63. Disponible en: http://article.sapub.org/10.5923.j.edu.20150502.02.html

Liberato, E. (2014). Avanços e retrocessos da educação em Angola. Revista Brasileira de Educação. 19 (59), 1003-1031. http://dx.doi.org/10.1590/S1413-24782014000900010

Ministério da Ciência e Tecnologia (s/f). Lista das Instituições de Investigação Científica e Desenvolvimento Tecnológico. Direcção Nacional de Investigação Científica, LuandaAngola. Disponible en: http://ciencia.ao/redes/rede_instituicoes.pdf

Ministério da Economia e Planeamento, (2018). Plano de Desenvolvimento Nacional PDN, (2018-2022). República de Angola. Disponible en: https://www.infoangola.com/attachments/article/4867/PDN\%202018-

2022_MASTER_vf_Volume\%201_13052018.pdf

Ministério do Ensino Superior, Ciência, Tecnologia e Inovação - MESCTI, (2019a). Relatório síntese das actividades desenvolvidas pelas Universidades Públicas de Angola no período de 2015-2018. Luanda, Conselho de Reitores das Universidades Públicas de Angola (CRUANG).

Ministério do Ensino Superior, Ciência, Tecnologia e Inovação - MESCTI, (2019b). Progressos, Fraquezas, Desafios e Oportunidades de Melhoria. Luanda, República de Angola.

Ministério do Ensino Superior, Ciência, Tecnologia e Inovação - MESCTI, (2018a). Estatuto Remuneratório da Carreira do Docente do Ensino Superior. Decreto Presidencial n. ${ }^{\circ}$ 280/18. República de Angola.

Ministério do Ensino Superior, Ciência, Tecnologia e Inovação - MESCTI, (2018b). Estatuto Remuneratório da Carreira do Investigador Científico. Decreto Presidencial n. ${ }^{\circ}$ 02/19. República de Angola.

Ministério do Ensino Superior, Ciência, Tecnologia e Inovação - MESCTI, (2018c). Estatuto da Carreira de Investigador Científico. Decreto Presidencial n. ${ }^{\circ}$ 109/19. República de Angola.

Ministério do Ensino Superior, Ciência, Tecnologia e Inovação - MESCTI, (2018d). Estatuto da Carreira Docente do Ensino Superior. Decreto Presidencial n. ${ }^{0}$ 191/18. República de Angola.

Ministério do Ensino Superior, Ciência, Tecnologia e Inovação - MESCTI, (2018e). Ajustamento dos vencimentos-base do Docente do Ensino Superior. Decreto Presidencial n. ${ }^{\circ}$ 299/18. República de Angola.

Paz Enrique, L. (2018). Actividad Editorial y Socializacion de la ciencia. Santa clara, Editorial Feijóo.

Pereira, A.; Casanova, M.; Pire, R. (2014) Estudio comparativo de la producción científica de las universidades públicas y privadas venezolanas. Compendium, vol. 17, núm. 32, 
julio, 2014, 55-77. Disponible en: https://www.redalyc.org/articulo.oa?id=88037910004

Plano Anual de Ciência, Tecnologia e Inonavação, - PLANCTI, (2014-2015). Decreto Presidencial n. ${ }^{\circ} 7 / 15$. Republica de Angola.

Política Nacional de Ciência, Tecnologia e Inovação - PNCTI, (2011). Decreto Presidencial n. ${ }^{\circ}$ 201/11. Republica de Angola.

Redalyc (2020). Criterios de evaluacion. Disponible en: https://www.redalyc.org/redalyc/ editores/reevaluacionCriterios.html

Sabino, C. (1992). El Proceso de Investigacion. Caracas, Ed. Panapo. Disponible en: http://c1130004.ferozo.com/instituto178/Academicos/Catedras/Verandi/CarlosSabinoElProcesodelaInvestigacion.pdf

Secretaria de Estado do Ensino Superior - SEES, (2015). Linhas Mestras Para A Melhoria da Gestão do Subsistema do Ensino Superior. Disponible en: https://planipolis.iiep.unesco.org/sites/planipolis/files/ressources/angola_linhas_mestras _subsistema_ensino_superior.pdf

Silva, E. A. (2016). Gestão do Ensino Superior em Angola: Realidades, Tendências e Desafios - Rumo à Qualidade. Luanda, Mayamba Editora.

Sistema Nacional de Ciência, Tecnologia e Inovação - SNCTI, (2011). Decreto Presidencial n. ${ }^{\circ}$ 224/11. Republica de Angola.

Sousa, J.; Rodrigues, M. J.; Pedro, A.; Manuel, A. (2018). Índice de Produção Científica dos Docentes do Instituto Superior de Ciências de Educação do Huambo. Telos. Revista de Estudios Interdisciplinarios en Ciencias Sociales. 20 (3), 450-467. https://doi.org/10.36390/telos203.04

Suárez Guava, L. (2017). Una breve historia de las revistas científicas en Colombia o la maldición de ser editor. Universitas Humanística, 83 (83). Disponible en: https://revistas.javeriana.edu.co/index.php/univhumanistica/article/view/18520

Tauchen, G.; Mendes, M.; Devechi, C. (2015). Garantia da qualidade e da avaliação: um estudo comparado sobre as decorrências do processo de Bolonha na comunidade dos países de língua portuguesa. Revista Espaço Pedagógico. 22 (2), 240-263. https://doi.org/10.5335/rep.v22i2.5567

Tessarini, G.; Saltorato, P. (2018). Impactos da Indústria 4.0 na organização do trabalho: Uma revisão sistemática da literatura. Revista Produção Online, 18 (2), 743-769. https://doi.org/10.14488/1676-1901.v18i2.2967

Teta, J. (2009). Educação superior em Angola. In Marília Costa Morosini (Ed.), Seminário Internacional de Educação Superior da Comunidade de Países de Língua Portuguesa, 30-34. Disponible en: http://www.pucrs.br/edipucrs/cplp/arquivos/teta.pdf 